MATEC Web of Conferences 51, 01004 (2016)

DOI: $10.1051 /$ matecconf/20165101004

C Owned by the authors, published by EDP Sciences, 2016

\title{
Vibration Characteristics of Tapered Roller Bearings with Roller Diameter error
}

\author{
Van-Canh Tong ${ }^{1}$ and Seong-Wook Hong ${ }^{1, a}$ \\ ${ }^{1}$ Department of Mechanical System Engineering, Kumoh National Institute of Technology, 61 Daehak-ro, Gumi, \\ Gyeongbuk, Republic of Korea 730-701
}

\begin{abstract}
This study investigates the vibration characteristics of tapered roller bearings with the presence of error in roller diameter. A general model for tapered roller bearing with five degree-of-freedom was adopted with taking into account the error in roller diameter. Then, the effect of one single errored roller and multiple errored rollers on the vibration behaviour of tapered roller bearing was investigated. Simulation results confirmed that the bearing vibration frequencies are equal to cage rotational frequency in the case of axial preloading, and multiple of cage rotational frequency in the case of combined loadings, irrespective of the error magnitude, the number or position of errored rollers, which, however, significantly affect the vibration amplitude of bearing.
\end{abstract}

\section{Introduction}

Rolling element bearings have been extensively used in rotating machineries to support load and ensure the rotational motion of the shaft. Vibration of rolling bearings is an important issue, which has a great attention in design and application of bearings. This is because the bearing vibration can considerably influence the overall performance of not only bearings but also the whole rotating systems containing bearings. Recently, the requirements for rotational vibration of bearings have become strict. Numerous research works have been implemented for detecting, monitoring, and controlling bearing vibration. A large number of studies have concerned about bearings with an assumption that no geometric error exists in the bearings. That assumption is, however, impractical since the geometric imperfections are often occurred in bearings during manufacturing process. This study deals with the vibration behaviour of tapered roller bearings (TRBs) with error in roller diameter.

The geometric error in rolling bearing can be classified into three groups, i.e. high frequency error as roughness, intermediate frequency error as waviness, and low frequency error as form error [1]. The most typical error source of roller is the form error, such as out of roundness and off-sized roller. The effect of off-sized roller, in particular, error in roller diameter on bearing vibration has been investigated by several researchers. However, most of them carried out investigation with regard to ball or cylindrical roller bearings [2-7]. Although TRBs are widely used in heavy load applications, the effect of geometric error on the performance of TRBs has not been sufficiently considered.

In this study, the effect of roller diameter error on the vibration characteristics of TRBs is investigated. A general five DOFs TRB model is employed with taking into account the error in roller

${ }^{\text {a }}$ Corresponding author : swhong@kumoh.ac.kr 


\section{MATEC Web of Conferences}

diameter. Then the effect of single and multiple errored rollers on the vibration of TRBs are investigated. The vibration of TRBs is evaluated using time and frequency domains, along with the demonstration of bearing centre orbits. Simulation is performed for a TRB subjected to axial preload and combined radial and axial loadings. The obtained results would be beneficial for tolerance design, manufacturing and loading of TRBs.

\section{Tapered roller bearing model}

Figure 1(a) shows the geometry of TRB along with external load and displacement vectors as

$$
\begin{aligned}
& \{F\}=\left\{\begin{array}{lllll}
F_{x} & F_{y} & F_{z} & M_{y} & M_{z}
\end{array}\right\}^{T} \\
& \{\delta\}=\left\{\begin{array}{llllll}
\delta_{x} & \delta_{y} & \delta_{z} & \gamma_{y} & \gamma_{z}
\end{array}\right\}^{T}
\end{aligned}
$$

The 5-DOF bearing model outlined by de Mul et al. [8] was used in this study. This model, however, is a simple quasi-static model, which does not take into account the time-varying position of the rolling elements. In order to establish a time-varying bearing model, the instantaneous angular position of a roller at time $t$ is defined as

$$
\phi_{j}(t)=\frac{2 \pi(j-1)}{Z}+\Omega_{m} t
$$
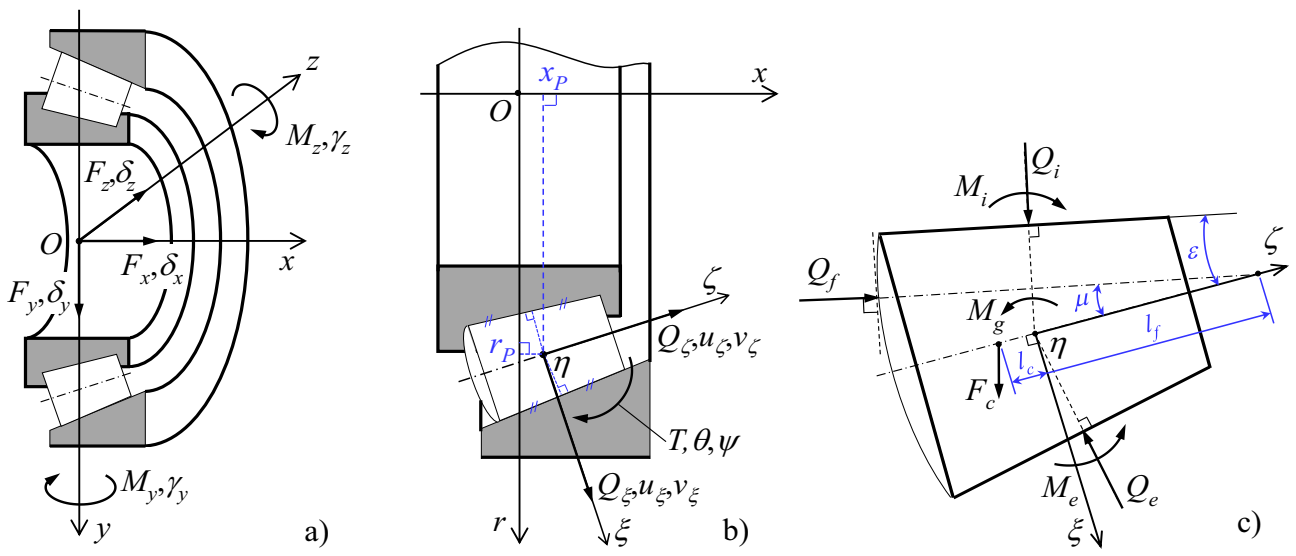

Figure 1. TRB geometry: a) TRB loading and displacements, b) TRB cross-section geometry at location angle $\phi_{j}(t)$ with roller and inner ring displacements $\left.\mathrm{c}\right)$ roller free-body diagram

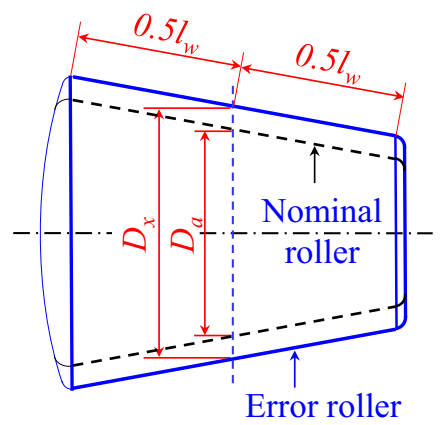

Figure 2. Nominal and errored roller geometry 
where $j$ and $Z$ represent the roller index and the total number of total rollers. $\Omega_{m}$ is the orbital speed of roller [9]. Figure 1(b) indicates the geometry of TRB cross-section at a particular roller location angle $\phi_{j}(t)$. The displacements of inner ring cross-section $\left\{u_{\kappa}\right\}=\left\{u_{r}, u_{z}, \theta\right\}^{T}$, can be calculated using transformation matrices $[K]$ and $\left[R_{\phi}\right]$ (see $\left.[8]\right)$

$$
\left\{u_{\kappa}\right\}=[K]\left(\left|R_{\phi}\right|\{\delta\}\right)
$$

Because the roller is displaced from its original position by a displacement vector $\left\{v_{\kappa}\right\}=\left\{v_{r}\right.$, $\left.v_{z}, \psi\right\}^{T}$, the roller and raceway contact loads $\left(Q_{i}, Q_{e}, M_{i}\right.$ and $\left.M_{e}\right)$ are generated as shown in Figure 1(c). These contact loads can be estimated using the slicing technique based on the contact compression between the roller and raceways [8]. The roller-flange contact force $Q_{f}$ is determined using the classical Herzian contact theory based on the contact between the spherical roller end and flat flange. The inertial loads acting on the roller including the centrifugal force $F_{c}$ and gyroscopic moment $M_{g}$ [9], are also considered in this study. Then the roller equilibrium equations can be obtained by considering two force equations about $\xi$ and $\zeta$ axes and a moment equation about $\eta$ axis as shown in Figure 1(c). By solving the roller equilibrium equations, the displacement of roller $\left\{v_{\kappa}\right\}$ can be determined, as well as the final value of roller-races contact loads. The roller contact loads must reflect the error in diameter. Figure 2 shows the geometry of the roller with and without error. The error magnitude is calculated as

$$
\delta_{D}=D_{x}-D_{a}
$$

where $D_{x}$ and $D_{a}$ are the errored and nominal diameters of roller (Figure 2).

Having obtained the inner ring contact loads at all rollers $\left\{Q_{\kappa}\right\}=\left\{Q_{r}, Q_{z}, T\right\}^{T}$, the inner ring equilibrium equations can be derived by summation of all inner ring contact loads and an external load $\{F\}$ as

$$
\{F\}+\sum_{j=1}^{z}\left\{\left[R_{\phi}\right]^{T}\left([K]^{T}\left\{Q_{\kappa}\right\}\right)\right\}_{j}=\{0\}
$$

Equation (6) is subsequently solved for the inner ring displacements $\{\delta\}$. All the dynamic equations of TRB including the roller equations and global equations of inner ring have been previously described in [6-7]. Because the roller and global equations are non-linear, the iterative technique should be used as a solution, for example Newton-Raphson method.

\section{Numerical results}

This section presents numerical results for the effect of roller diameter error on the TRB vibration behaviour. A sample TRB (30212-A) with its basic parameters given in Table 1 was used for investigation. The rotational speed of TRB is selected at $1000 \mathrm{rpm}$ throughout the paper.

Table 1. Basic parameters of TRB 30212-A.

\begin{tabular}{|c|c|c|c|c|c|}
\hline Parameter & Symbol & Value & Parameter & Symbol & Value \\
\hline Bore diameter & $d[\mathrm{~mm}]$ & 60 & Number of rollers & $z$ & 19 \\
\hline Outside diameter & $D[\mathrm{~mm}]$ & 110 & Pitch diameter & $d_{m}[\mathrm{~mm}]$ & 84.12 \\
\hline Total width & $B[\mathrm{~mm}]$ & 23.75 & Nominal roller mean diameter & $D_{a}[\mathrm{~mm}]$ & 13 \\
\hline
\end{tabular}

\subsection{Single errored roller}




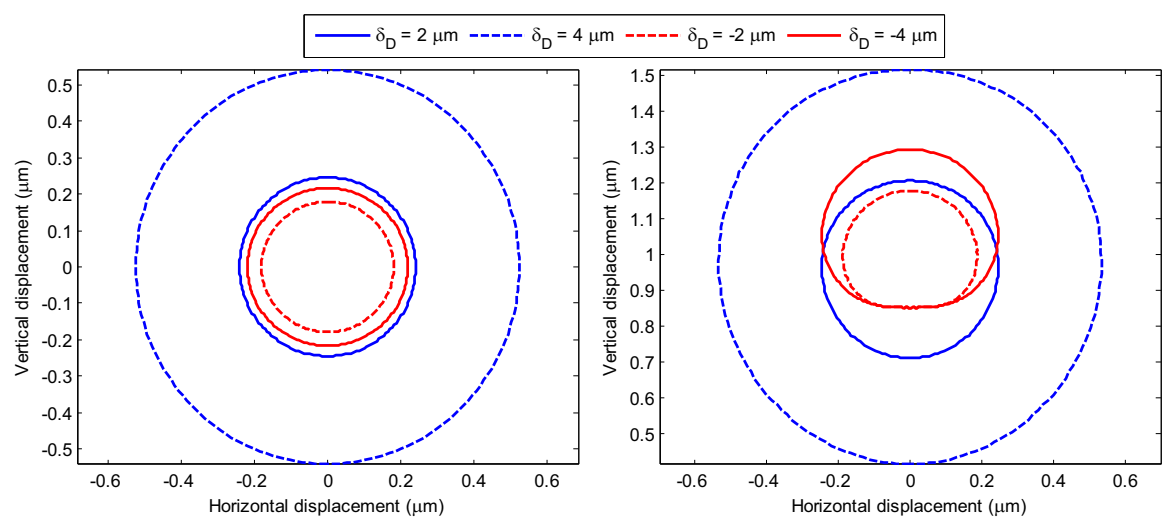

Figure 3. TRB centre orbits with single errored roller: a) $F_{x}=1000 \mathrm{~N}$, b) $F_{x}=F_{y}=1000 \mathrm{~N}$
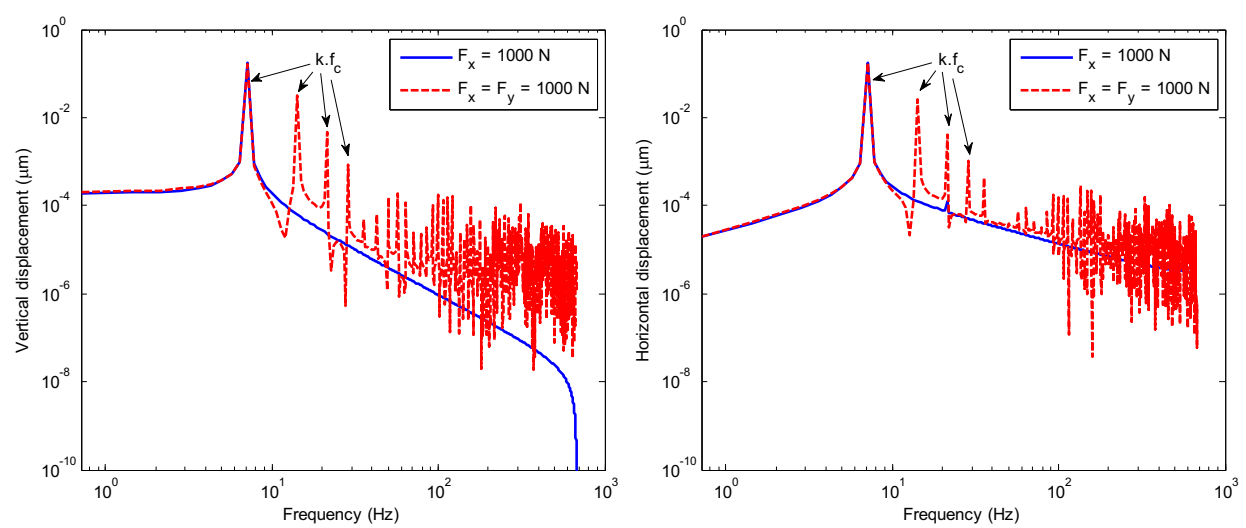

Figure 4. Spectrums of TRB displacements with errored roller with the error magnitude of $\delta_{D}=2 \mu m$

Figure 3 illustrates the centre orbits of the TRB with one errored roller. It is seen that the magnitude of the centre orbits is increased with increasing the error magnitude. In the case of axial preload TRB, the bearing centre trajectories are remained circular regardless of error magnitude. However, the trajectories are no longer circular when the TRB is loaded with combined radial and axial loads. Figure 4 shows the frequency spectrums of vertical and horizontal displacements of TRB. The TRB under only axial preload shows only single dominant peak frequency equal to the cage rotational frequency $\left(f_{c}\right)$. However, under combined loads, the roller error excites many vibration frequencies corresponding to multiples of the cage frequency $\left(k . f_{c}\right)$

\subsection{Multiple errored rollers}

\subsubsection{Identical error magnitude}

Figure 5 indicates the centre orbits of TRB with different combination of errored rollers. The TRB has two errored rollers whose error magnitude is selected $2 \mu \mathrm{m}$. From Figure 5, the set of errored rollers at $\# 1$ and \#2 shows the highest radial vibration, and the next orders are the sets of errored roller at \#1 and $\# 4, \# 1$ and $\# 8$, and \#1 and \#10. Here the number represents the consecutive order among the rollers in the TRB. This is attributed by the increase of the distance angle between the errored rollers. Therefore, the vibration of TRB can be reduced when errored rollers are distributed around the bearing circumference. 
Figure 6 demonstrates the frequency spectrums of TRB with errored rollers at \#1 and \#2. The frequency components are quite similar to those in the case of single errored roller shown in Figure 4 with its dominant frequencies at multiple harmonics of $f_{c}$. It means that the number, position and magnitude of error rollers do not significantly affect the frequency component of TRB vibration.

\subsubsection{Different error magnitude}

Vibration of TRB is evaluated for the case when there exist two errored rollers with different error magnitude of 2 and $-2 \mu \mathrm{m}$. Figure 7 illustrates the centre orbits of TRB with different combinations of errored rollers. From Figure 7, the vibration of TRB shows a behaviour contrary to that for the case of identical errored rollers shown in Figure 5. The largest centre orbit is found when the errored rollers are at \#1 and \#10, whereas the smallest one is for the case of errored rollers at \#1 and \#2.
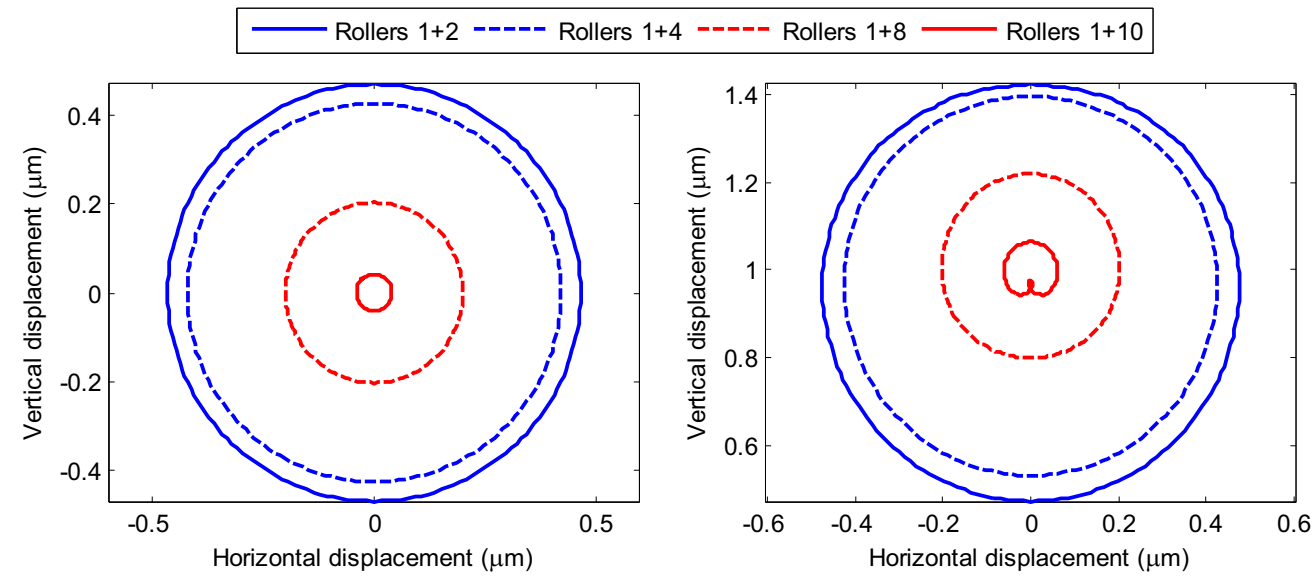

Figure 5. TRB centre orbits with two errored rollers with error magnitude of $2 \mu \mathrm{m}$ : a) $F_{x}=1000 N$, b) $F_{x}=F_{y}=1000 \mathrm{~N}$
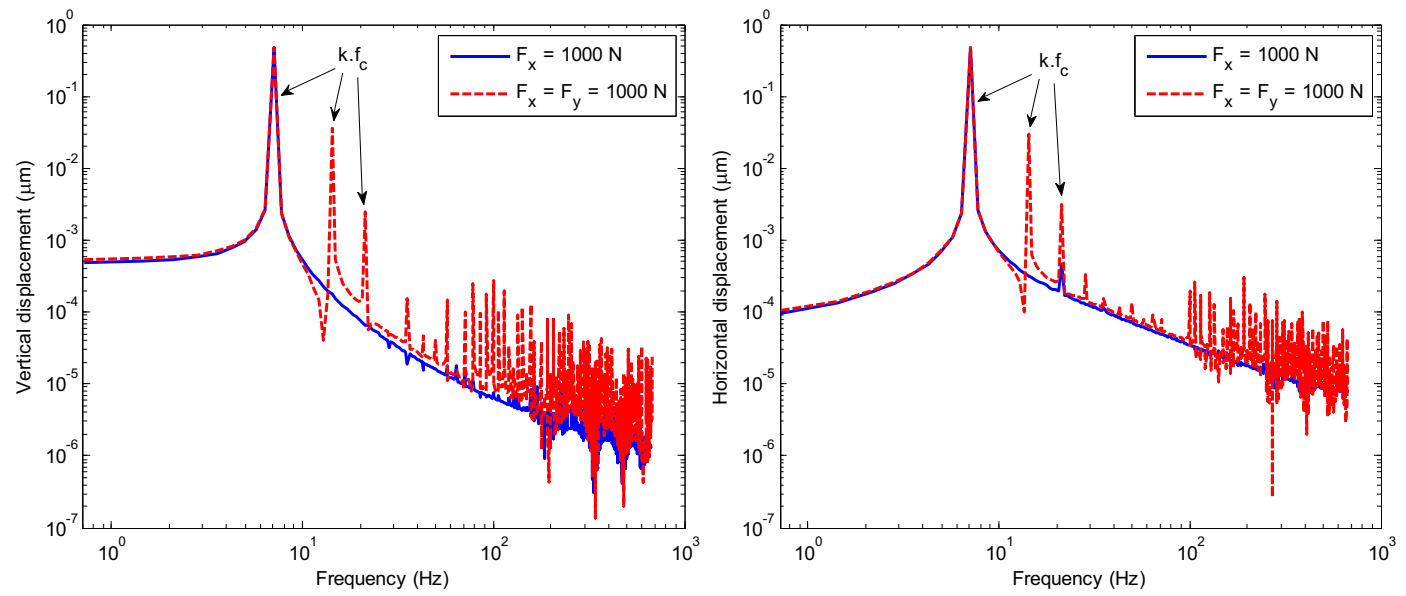

Figure 6. Spectrums of TRB displacements with errored rollers at \#1 and \#2 with error magnitude of $2 \mu \mathrm{m}$ 

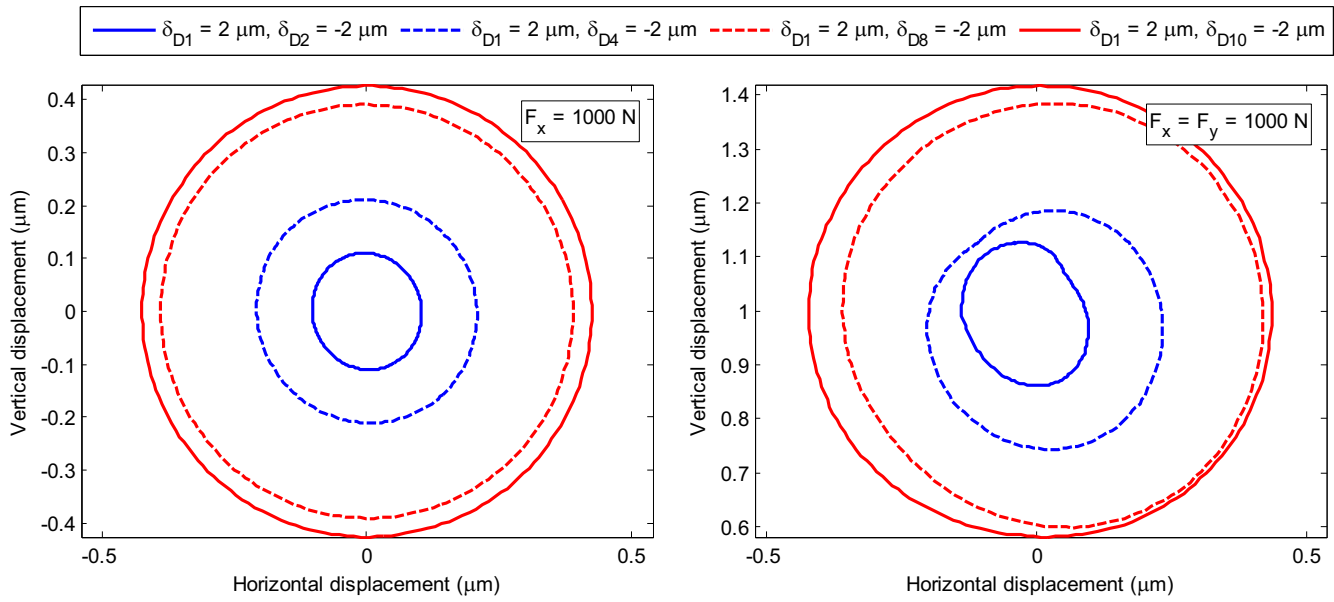

Figure 7. TRB centre orbits with two errored rollers with different error magnitudes, $2 \mu \mathrm{m}$ and $-2 \mu \mathrm{m}$

\section{Conclusion}

From the simulation results for vibration response of TRB with errored rollers, following conclusions are obtained:

1. The roller diameter error excites the TRB at the cage rotational frequency in the case of axial preload, at multiple harmonics of cage rotational frequency in the case of combined axial and radial loads.

2. Increasing roller error magnitude significantly increases the vibration amplitude of TRB. However, roller error magnitude does not affect the frequency components of TRB, likewise the number and position of errored rollers.

\section{Acknowledgement}

This research was financially supported by Daegyeong Institute of Regional Program Evaluation.

\section{References}

1. X. D. Zhang, C. Zhang, B. Wang. American Soc. Precis. Eng. (1999)

2. L. D. Meyer, F. F. Ahlgren, B. Weichbrodt. J. Mech. Des 102, 2 (1980)

3. P. K. Gupta. ASME J. Tribol. 110, 1 (1988)

4. S. Noguchi, K. Ono. Precis. Eng. 28, 2004

5. G. Chen, F. Mao, B. Wang. Proc. IMechE. Part J: Part J: J. Eng. Tribol. 226, 8 (2012)

6. S. Yu, D. Wang, H. Dong, B. Wang. Proc. IMechE. Part C: J Mech. Eng. Sci. 227, 11 (2013)

7. P. Ji, Y. Gao, F. Ma and Q. An. Proc. IMechE. Part J: J. Eng. Tribol. 229, 6 (2015)

8. J. M. de Mul, J. M. Vree, D. A. Maas. ASME J. Tribol. 111, (1989)

9. V. C. Tong, S. W. Hong. Proc. IMechE. Part C: J Mech. Eng. Sci. 230, 2 (2016) 